\title{
K-means and associated cuckoo based hierarchy optimization for document categorization
}

\author{
Chandni Sikarwar ${ }^{*}$, Kailash Patidar ${ }^{2}$ and Rishi Kushwah ${ }^{3}$ \\ M.Tech Scholar, Department of Computer Science, School of Engineering, Sri Satya Sai University of Technology \\ $\&$ Medical Sciences, Sehore, Madhya Pradesh, India ${ }^{1}$ \\ Professor and HOD, Department of Computer Science, School of Engineering, Sri Satya Sai University of \\ Technology \& Medical Sciences, Sehore, Madhya Pradesh, India ${ }^{2}$ \\ Assistant Professor, Department of Computer Science, School of Engineering, Sri Satya Sai University of \\ Technology \& Medical Sciences, Sehore, Madhya Pradesh, India ${ }^{3}$
}

\section{(C)2018 ACCENTS}

\begin{abstract}
In this paper a hybrid algorithm for text data categorization has been proposed. There are four different classifiers of filtrations have been used. First classifier is numeric classifier $(\mathrm{NC}) . \mathrm{NC}$ is used for the numeric value classification and removal. Second classifier is separator classifier (SC). SC is used for the delimiter value classification and removal. Third classifier is all classifier (AC). AC is used for the grammar classification and removal. Last classifier is manual data classifier (MDC). MDC is used for the manual data value classification and removal. Then the associated cuckoo search optimization (CSO) based hierarchy optimization has been applied on the obtained data. Overall accuracy obtained by our approach is approximately $95 \%$.
\end{abstract}

\section{Keywords}

K-means, Associated cuckoo based hierarchy optimization, NC and SC, MDC.

\section{Introduction}

In current scenario proper document retrieval is a major concern. Retrieving the appropriate document is important for efficient retrieval of document. It is helpful in appropriate retrieval and categorization.

In certifiable exigency, intentionality producer's bear unendingly reasonably add to conflicted goals and a far reaching satisfies with contrastive sprinter options [1-5]. The multi-basis improving additionally give a battle sooner rather than later. Its includes traverse thorough spaces like the outline space, consolidating the characterizing factors of the competitor arrangements, and the expectation space, constituting the mapping of every hopeful answer for the various target capacities values [6]. The last is where optimality is getting going, trade-offs are investigated, and choices are ordinarily come to.

So there is the need of arrangement in light of different choice criteria which can be heuristic, it will be conceivable by client characterized imperatives and numerous specific requirements.

*Author for correspondence

297
It can be smarter to locate a legitimate bunched approach to arrange the records, at that point apply some characterization criteria which will be fulfilled some edge an incentive to give the compelled method for these issue. It can be accomplished through affiliation lead mining [7], we can utilize apportioning system additionally in light of the fact that it can diminish the looking time and upgrade the seeking ability $[8,9]$.

For characterization we can utilize affiliation run mining with some grouping procedures like k-means and fuzzy c-means, it will be a superior choice [10]. At that point we can upgrade it utilizing a few streamlining systems like ant colony optimization (ACO), particle swarm optimization, Mimetic calculation etc. [11-13]. Subset superset dividing can be utilized for parcelling and better grouping [14].

There is an exponential increment in the quantity of advanced information and content records. Accordingly, it is exceptionally hard to sort out these expansive accumulation of content reports in an successful way and finding fascinating data or examples $[15,16]$ has turned into an essential undertaking. To accelerate the looking procedure for 
comparable reports for finding required archives, record grouping is a strategy generally utilized. Grouping out an extensive arrangement of records into various comparative groups [17]. Along these lines, the records in a similar bunch are more like each other than to records in another group. Preprocessing is done to change over the words to their base frame, to expel stop words, copy words before applying vector space model to the content records. The separations between records are estimated utilizing closeness measures like Cosine, Jaccard [18] and so on. At that point the bunching calculations are connected till required number of groups is framed. There are two normal grouping calculations. Dividing calculations in which groups are registered straightforwardly.

In this paper an efficient approach has been proposed for document clustering.

\section{Literature review}

In 2011, Zuhtuogullari and [19] observe that an extendable and improved item set generation approach has been constructed and developed for mining the relationships of the symptoms and disorders in the medical databases. The algorithm of the developed software finds the frequent illnesses and generates association rules using Apriori algorithm. The developed software can be usable for large medical and health databases for constructing association rules for disorders frequently seen in the patient and determining the correlation of the health disorders and symptoms observed simultaneously.

In 2010, Yang et al. [20] suggest that the SOM has main disadvantage of the need to know the number and structure of neurons prior to training, which are difficult to be determined. Several schemes have been proposed to tackle such deficiency. Examples are growing/expandable SOM, hierarchical SOM, and growing hierarchical SOM. These schemes could dynamically expand the map; even generate hierarchical maps, during training. Encouraging results were reported. Basically, these schemes adapt the size and structure of the map according to the distribution of training data. That is, they are datadriven or data oriented SOM schemes. In this work, a topic-oriented SOM scheme which is suitable for document clustering and organization will be developed. Their proposed SOM will automatically adapt the number as well as the structure of the map according to identified topics. Unlike other dataoriented SOMs, our approach expands the map and generates the hierarchies both according to the topics and their characteristics of the neurons. The preliminary experiments give promising result and demonstrate the plausibility of the method.

In 2013, Yang et al. [21] two major deficiencies of classical SOM are the need of predefined map structure and the lack of hierarchy generation. Several approaches have been devised to tackle these deficiencies. They suggest that both structural and topical constraints which specified by the user could be used to guide the learning process. Preliminary experiments demonstrate improvements over previous algorithm on text categorization task.

In 2016, Pradip and Patil [22] suggested that the traditional clustering algorithms have some problems like instability of clusters, complexity and sensitivity. They have implemented a hierarchical and fuzzy relational eigenvector centrality-based clustering algorithm. The exploratory result demonstrates that hierarchical grouping will be valuable calculation for content records and gives better outcomes.

In 2016, Harish et al. [23] proposed a novel text categorization method. It is based on modified support vector clustering (SVC). The principle disadvantage of customary SVC is that it regards unclassified reports as anomalies. They have used fuzzy c-means (FCM) for this drawback. The changed (SVC-FCM) is connected to order message records. They have used FCM on the unclassified documents. To assess the execution of the proposed strategy, they led probes standard 20-NewsGroup dataset.

In 2017, Popat et al. [24] a test investigation of closeness based technique, HSC for estimating the similitude between information protests especially message archives is presented. It additionally gives a calculation which has an incremental approach and assesses group resemblance between archives that prompts much enhanced outcomes over other conventional techniques. It likewise centers on the determination of fitting likeness measure for examining comparability between the records.

In 2016, Dou and Liu [25] suggested that the visual text analysis is important in the real world scenario. They have proposed visual metaphors for visual text analysis. Thus, measures and benchmark datasets would significantly help the exploration network assess, arrange, furthermore, think about visual content examination frameworks. 
In 2016, Kohana et al. [26] suggested a distributed calculation scheme. It is helpful in scoring relationship among documents. This plan sorts reports by utilizing a calculation which computes a score and incentive for the connection between a classification and a word in a record. The more drawn out estimation time progresses toward becoming when expanding the quantity of reports. Subsequently, our plan utilizes different machines. An ace hub isolates a record set into a few subsets, and it circulates them to every estimation hubs. Utilizing this circulated estimation makes the figuring time short, and furthermore makes the memory utilization low.

In 2015, Nema and Sharma [27] proposed a feature optimization based multi-label text categorization. The procedure of highlight improvement is finished by subterranean insect settlement improvement. Their approach is based on ant colony optimization. For the procedure of order utilized bunch mapping characterization strategy. The element enhancement process lessens the loss of information amid the change of highlight mapping amid the order. For the approval of proposed calculation utilized some standard dataset, for example, site page information, medicinal inquiry information and RCV1 dataset. Our exact assessment demonstrates that proposed calculation is superior to fluffy pertinence procedure.

In 2015, Bide and Shedge [28] suggested that the need of faster categorization of documents for the forensic investigation. Along these lines, there is a need to discrete different accumulations of archives into comparable ones through bunching. Indicating number of groups is obligatory in existing apportioning calculations and the yield is absolutely reliant on given info. Over bunching is the significant issue in archive grouping. Their proposed calculation takes contribution as keywords found after extraction and takes care of the issue of over grouping by isolating the records into little gatherings utilizing divide and vanquish strategy. Test comes about demonstrated that exactness of proposed calculation is high contrast with existing calculation as far as measure and time complexity.

In 2016, Wandabwa et al. [29] suggested that the content order involves settling on a choice on regardless of whether a record has a place with an arrangement of pre-indicated classes of different records. The key component of this calculation lies in the closeness estimation rule that is equipped for distinguishing neighbours of a specific archive to high correctness's. The main disadvantage of this approach is in the weighting of all highlights to decide the separation among the reports being referred to. Their approach has the improved performance as compared to the classical KNN.

\section{Proposed work}

A hybrid k-means and associated cuckoo based hierarchy optimization for document categorization have been proposed in this paper. Flowchart shown in Figure 1 provides us the detail exploration of our approach. Our approach is divided in four different parts. In the first step the data filtration have been applied. Data filtration is helpful in text data filtration. It is helpful in the classification of word and provides us the word rank or weight.

There are four different classifiers of filtrations have been used. First classifier is numeric classifier (NC). $\mathrm{NC}$ is used for the numeric value classification and removal. Second classifier is separator classifier (SC). SC is used for the delimiter value classification and removal. Third classifier is all classifier (AC). $\mathrm{AC}$ is used for the grammar classification and removal. Last classifier is manual data classifier (MDC). MDC is used for the manual data value classification and removal.

Then the gathered data which is the combination items with the association rules along with the weight frequency have been send for the further processing. The removed classified data has been added to the database for the preparation of advance dataset for the next data categorization. Then the associated cuckoo search optimization (CSO) based hierarchy optimization has been applied on the obtained data. The algorithm for the processing is shown below. In this phase the data is clustered according to the $\mathrm{k}$ means algorithm. Ten clusters have been considered in our approach. For each categorization a minimum threshold has been applied and the algorithm has been applied separately for each cluster for the rank categorization. We have applied maximization and minimization both cases with CSO algorithm.

\section{Result analysis}

The overall categorization filters 10 best results based on the minimization and maximization. Different category of data has been classified through our approach which shows the potential of our approach. Figure 2 shows the result comparison based on the Yang et al. [21]. 
Sikarwar et al.

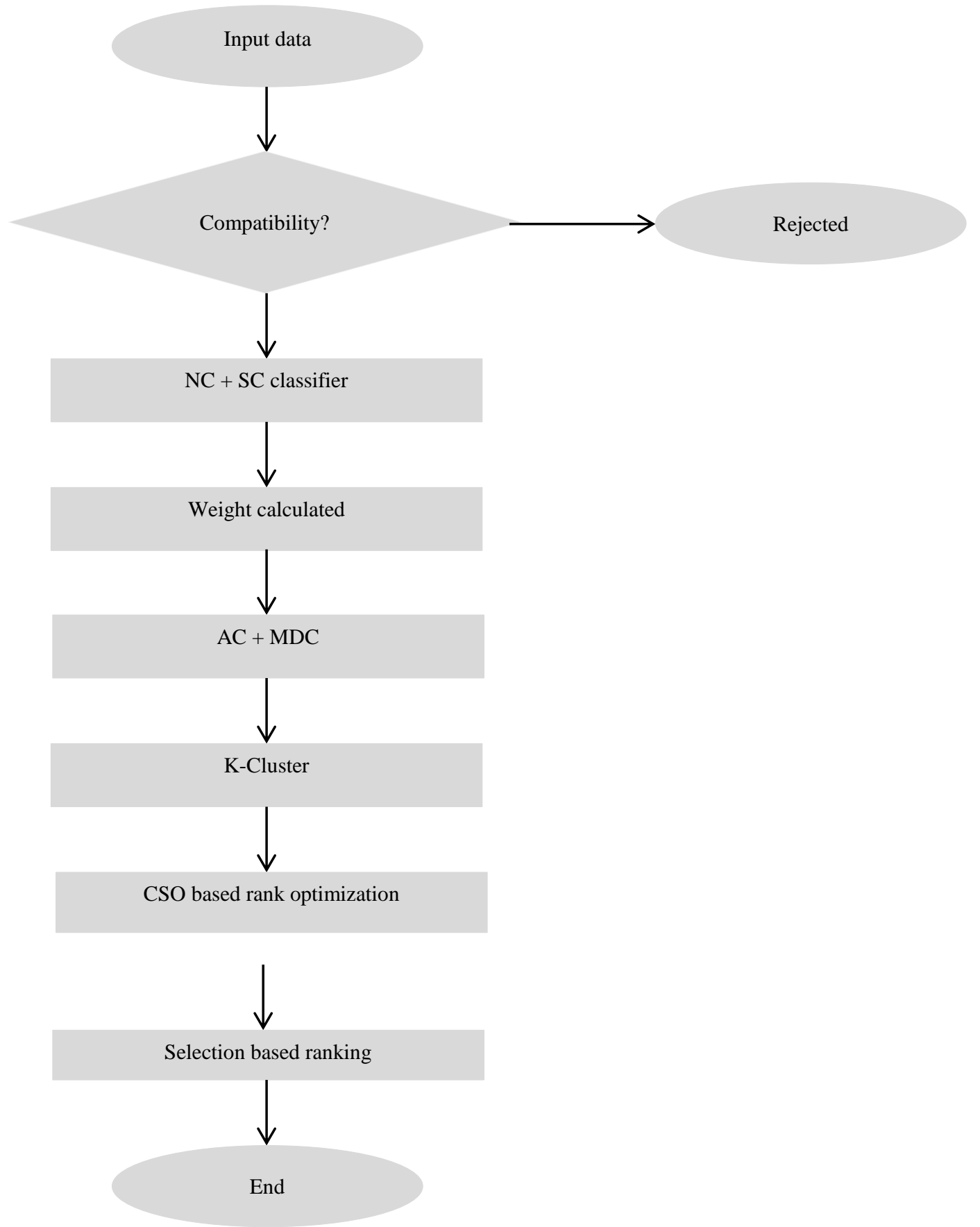

Figure 1 Flowchart for proposed work 


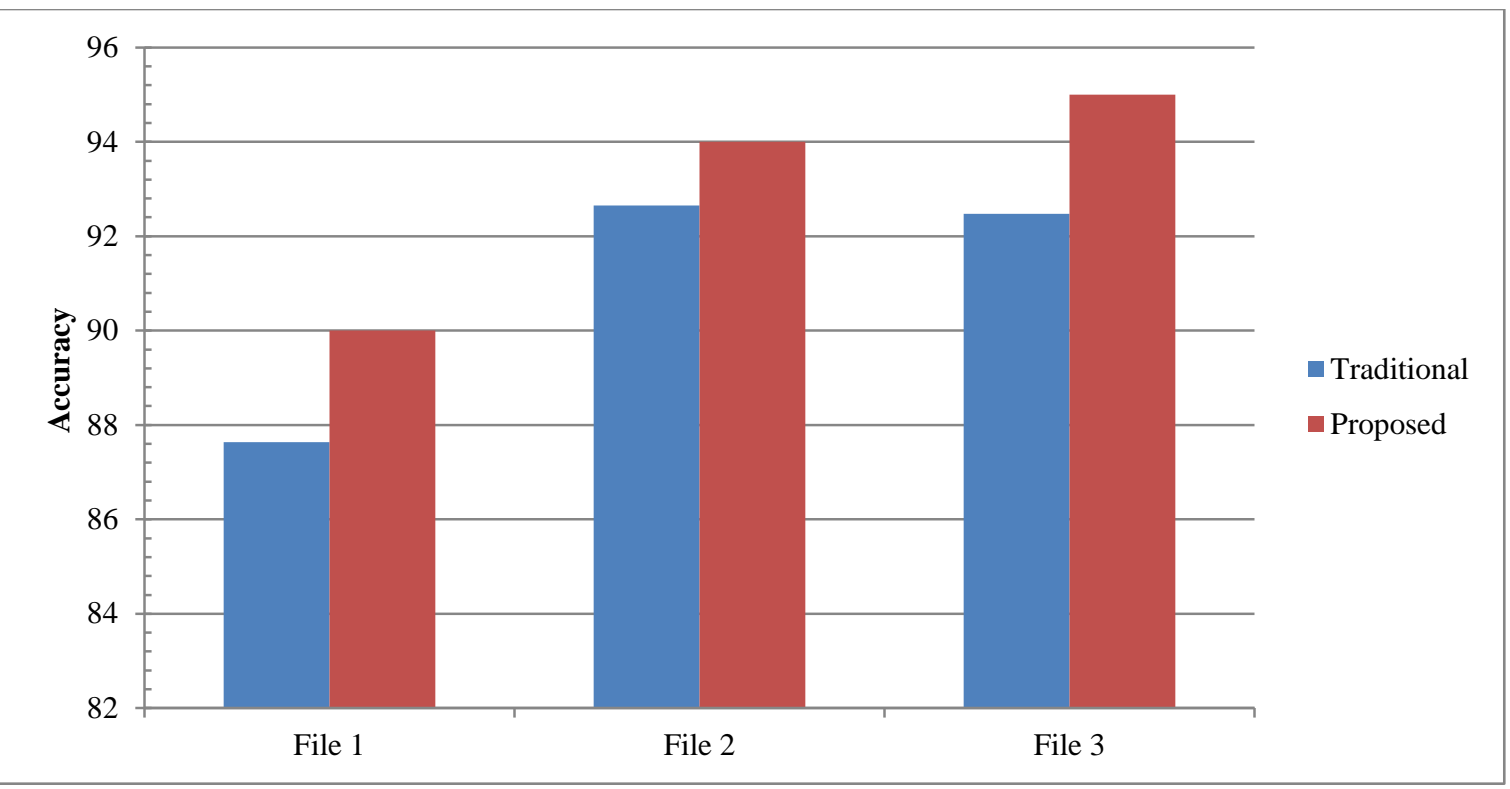

Figure 2 Performance comparison based on the Yang et al. [21]

\section{Conclusion}

In this paper a hybrid algorithm for text data categorization has been presented. In our approach we have used three different approaches in a single framework. There are four different classifiers of filtrations have been used. First classifier is numeric classifier (NC). NC is used for the numeric value classification and removal. Second classifier is separator classifier (SC). SC is used for the delimiter value classification and removal. Third classifier is all classifier (AC). AC is used for the grammar classification and removal. Last classifier is manual data classifier (MDC). MDC is used for the manual data value classification and removal. Then we have proposed associative cluster to categorize the text data and then apply cuckoo search algorithm for finding the optimize rank in the related class hierarchy. For experimentation we have applied our approach on different data and achieve better results in comparison to the previous methods.

\section{Acknowledgment}

None.

\section{Conflicts of interest}

The authors have no conflicts of interest to declare.

\section{References}

[1] Azizah A, Abraham J. Content analysis and exploratory factor analysis of relationship goals among young adults: converging data from instagram and offline surveys. International Journal of Advanced Computer Research. 2017; 8(34):11-34.
[2] Rauber A, Dittenbach M, Merkl D. Towards automatic content-based organization of multilingual digital libraries: an English, French, and German view of the Russian information agency novosti news. In third allRussian conference digital libraries: advanced methods and technologies 2001.

[3] Rauber A, Merkl D, Dittenbach M. The growing hierarchical self-organizing map: exploratory analysis of high-dimensional data. IEEE Transactions on Neural Networks. 2002; 13(6):1331-41.

[4] Bagajewicz M, Cabrera E. Pareto optimal solutions visualization techniques for multiobjective design and upgrade of instrumentation networks. Industrial \& Engineering Chemistry Research. 2003; 42(21):5195203.

[5] Berger W, Piringer H, Filzmoser P, Groller E. Uncertainty-aware exploration of continuous parameter spaces using multivariate prediction. Computer Graphics Forum. 2011; 30(3):911-20.

[6] Beume N, Naujoks B, Emmerich M. SMS-EMOA: Multiobjective selection based on dominated hypervolume. European Journal of Operational Research. 2007; 181(3):1653-69.

[7] Dubey AK, Shandilya SK. A novel J2ME service for mining incremental patterns in mobile computing. In international conference on advances in information and communication technologies 2010 (pp. 157-64). Springer, Berlin, Heidelberg.

[8] Shrivastava P, Gupta H. A review of density-based clustering in spatial data. International Journal of Advanced Computer Research. 2012; 2(5):200-2.

[9] Chen K, Liu L. A random rotation perturbation approach to privacy preserving data classification. In proceedings of international conference on data mining 2005 (pp. 1-12). 
[10] Liang SC, Lee YC, Lee PC. The application of ant colony optimization to the classification rule problem. In international conference on granular computing 2011 (pp. 390-2). IEEE.

[11] Sadh AS, Shukla N. Association rules optimization: a survey. International Journal of Advanced Computer Research. 2013; 3(9):111-5.

[12] Modiri A, Kiasaleh K. Permittivity estimation for breast cancer detection using particle swarm optimization algorithm. In annual international conference of the engineering in medicine and biology society 2011 (pp. 1359-62). IEEE.

[13] Liu Y, Chung YY. Mining cancer data with discrete particle swarm optimization and rule pruning. In international symposium on IT in medicine and education 2011 (pp. 31-4). IEEE.

[14] Dubey AK, Dubey AK, Agarwal V, Khandagre Y. Knowledge discovery with a subset-superset approach for mining heterogeneous data with dynamic support. Conseg-2012 (pp.1-6). IEEE.

[15] Srihari S, Leedham G. A survey of computer methods in forensic handwritten document examination. In eleventh international graphonomics society conference 2003 (pp. 278-81).

[16] Oppliger R, Rytz R. Digital evidence: dream and reality. IEEE Security \& Privacy. 2003; 99(5):44-8.

[17] Mehrbod A, Zutshi A, Grilo A. A vector space model approach for searching and matching product ecatalogues. In proceedings of the eighth international conference on management science and engineering management 2014 (pp. 833-42). Springer, Berlin, Heidelberg.

[18] Bai VM, Manimegalai D. An analysis of document clustering algorithms. In international conference on communication control and computing technologies 2010 (pp. 402-6). IEEE.

[19] Zuhtuogullari K, Allahverdi N. An improved itemset generation approach for mining medical databases. International symposium on innovations in intelligent systems and applications 2011 (pp. 39-43). IEEE.

[20] Yang HC, Lee CH, Ke KL. TOSOM: a topic-oriented self-organizing map for text organization. International Journal of Computer and Information Engineering. 2010; 4(5):1013-7.

[21] Yang $\mathrm{HC}$, Lee $\mathrm{CH}$, Wu CY. Incorporating user constraints into topic-oriented self-organizing maps. In symposium on foundations of computational intelligence 2013 (pp. 91-7). IEEE.
[22] Pradip KG, Patil DR. Summarization of sentences using fuzzy and hierarchical clustering approach. In symposium on colossal data analysis and networking 2016 (pp. 1-7). IEEE.

[23] Harish BS, Revanasiddappa MB, Kumar SA. A modified support vector clustering method for document categorization. In international conference on knowledge engineering and applications 2016 (pp. 1-5). IEEE.

[24] Popat SK, Deshmukh PB, Metre VA. Hierarchical document clustering based on cosine similarity measure. In international conference on intelligent systems and information management 2017 (pp. 1539). IEEE.

[25] Dou W, Liu S. Topic-and time-oriented visual text analysis. IEEE Computer Graphics and Applications. 2016; 36(4):8-13.

[26] Kohana M, Sakaji H, Kobayashi A, Okamoto S. A distributed calculation scheme for contents categorization. In international conference on advanced information networking and applications 2017 (pp. 614-20). IEEE.

[27] Nema P, Sharma V. Multi-label text categorization based on feature optimization using ant colony optimization and relevance clustering technique. In international conference on computers, communications, and systems 2015 (pp. 1-5). IEEE.

[28] Bide P, Shedge R. Improved document clustering using k-means algorithm. In international conference on electrical, computer and communication technologies 2015 (pp. 1-5). IEEE.

[29] Wandabwa H, Zhang D, Sammy K. Text categorization via attribute distance weighted $\mathrm{k}$ nearest neighbor classification. In international conference on information technology 2016 (pp. 2258). IEEE.

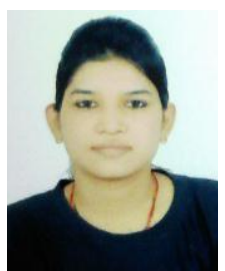

Chandni Sikarwar had completed her $\mathrm{BE}$ from Alpine Institute of Technology, Ujjain, M.P. in 2012 in the Department of Computer Science and Engineering. Currently she is pursuing M.Tech in Computer Science from SSSITS, Sehore.

Email: chandni.sikarwar90@gmail.com 